\title{
Sensor Based Mobile Land Slide Detector
}

\author{
Pradnya Garad \\ PG Student, E \& TC Department, G.H Raisoni College of engineering, Ahmednagar, Pune University
}

\begin{abstract}
Mobile land slide detector based on sensor network for landslide detection. The whole system was validated by the issuance of a landslide warning in the month of July (last monsoon 3season), which facilitated pre-emptive action by the local government and community to prevent loss of human life. In this system rain sensor, soil moisture sensor, flex sensor, accelerometer sensor these sensors are used for detection of landslide.
\end{abstract}

Keywords: sensor network, rainfall monitoring

\section{Introduction}

The Advent of sensor networks and wireless technologies offers the capability of quick capture, processing, and transmission of critical disaster data in real-time from inaccessible sites, incurring minimum maintenance as well. India faces rainfall-induced landslides every year with a large threat to human life and an annual loss of U.S. \$400 million.

In this system four sensor network use to landslide detection. Rain sensor senses rainfall, soil moisture senses soil moisture contain, to sense the movement of earth flex sensor \& accelerometer sensor.These sensors ADC values are given to arduino processor (inbuild ADC).GPS system is to locate the continuous location of landslide.GSM system through all sensor reading always send to save number.

Rain sensor reads high rain then it increases soil moisture \& also the movement of soil done in this season. This is the landslide situation. After detection of it will given to SMS to saved number.

\section{Aim of Project}

This system informs emergency services via sms or email before landslides.

1) We can monitor no of such sites from single host computer.

2) Provides graphical representation of day to day varying parameter

\section{Relation to Previous Work}

Sensor networks has been an active research area for almost a decade. Environmental monitoring, as well as energy conservation have been significant priorities in many of these studies. Some of the earliest schemes propose keeping sensors on only when there is data [1]. Another popular technique to reduce data is by aggregation and fusion [2], [3]. Sensor networks for (i) slip surface monitoring have been proposed by Terzis, Anandarajah, Moore, and Wang, (ii) flood monitoring by Kuang, Quek, and Mallej, (iii) active volcano monitoring by Werner-Allen, Lorinez,Welsh, Marcillo, Johnson, Ruiz, and Lees, (iv) forest fire monitoring by Hefeeda and Bagheri, (v) drought monitoring by Kung and Hua, (vi) wireless soil ecology monitoring by Musaloiu, Terzis, Szlavecz, Szalay, Cogan, and Gray, (vii) coal mines monitoring by Unnikrishnan et al. However, there are no studies that have systematically quantified the effectiveness of different types of sensors for monitoring disasters, particularly, landslides. Further, there are no studies reporting adequate amounts of data from real field deployments. This project's distinguishing contribution is to present studies from significant amounts of data collected from a real world field deployment that has been kept operational for sufficiently long duration amidst inclement weather conditions. This analyze each sensor for its data generated and energy consumption, then compute its sustainability for the entire monsoon season, and correlate the predictability of landslides from the sensor data.

\section{Sensor Networks for Landslide Detection and Monitoring}

When we embarked on developing a sensor network to monitor and detect landslides, our first task was to determine which sensor (or sensors) to use. Since landslides are commonly triggered by intense downpours or prolonged medium intensity rainfall, our first choice was a tipping bucket type rain gauge as the sensor(fig 1)

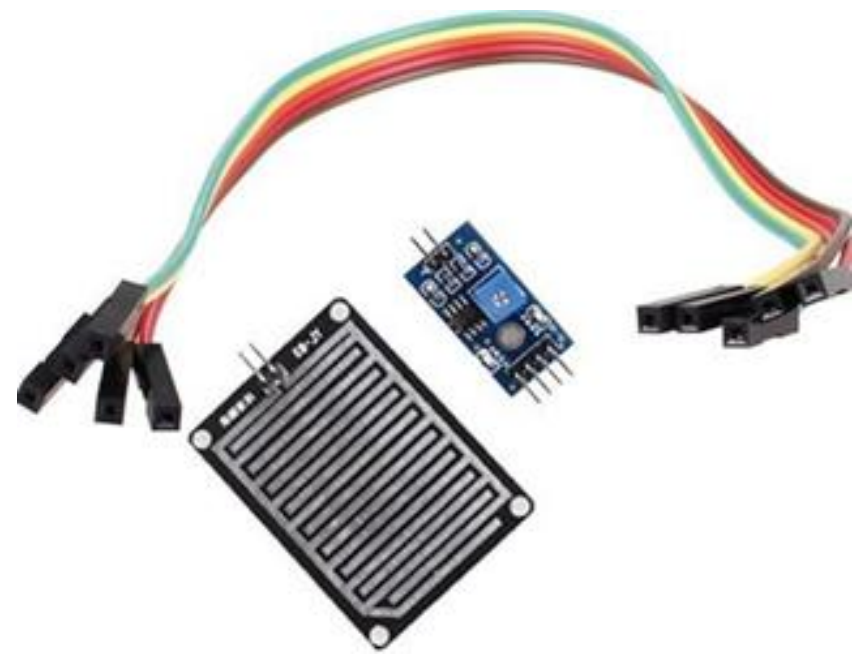

Figure 1: Rain sensor

Rain sensor continuously senses rainfall. Rain sensor is resistive type sensor if rain increases resistance of rain sensor increases \& this is the ADC value of rain sensor.[1]

Soil moisture sensor is probe type sensor (fig 2). 


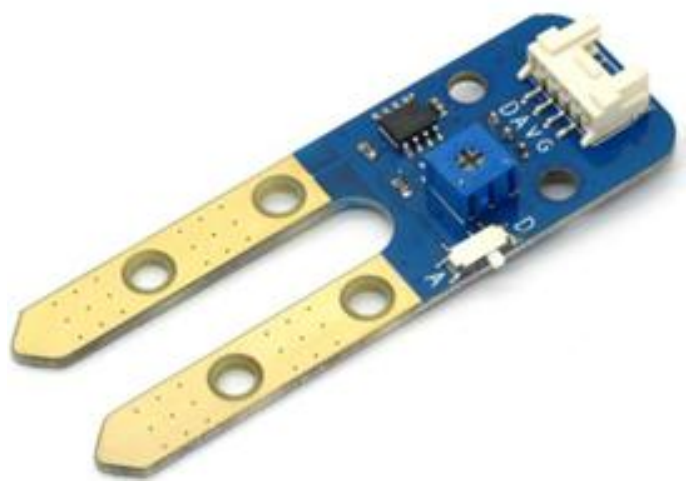

Figure 2: Soil moisture sensor

Soil moisture sensor probes are continuously deep into soil. If soil moisture increases resistance of sensor increases this is the ADC value of controller. [2]

Flex sensor

Flex Sensor 2.2"(fig 3) RoHS Compliant Description: A simple flex sensor 2.2" in length. As the sensor is flexed, the resistance across the sensor increases. The resistance of the flex sensor changes when the metal pads are on the outside of the blend.

Accelerometer sensor(The ADXL335 )is a small, thin, low power, complete 3-axis accelerometer with signal conditioned voltage outputs. The product measures acceleration with a minimum full-scale range of $\pm 3 \mathrm{~g}$. It can measure the static acceleration of gravity in tilt-sensing applications, as well as dynamic acceleration resulting from motion, shock, or vibration.

\section{Controller}

In this project arduino uno 32 bit microcontroller[14] is used .It has following characteristic-

1) 32 kByte Flash Memory

2) 1 kByte EEPROM

3) 2 kByte SRAM

4) $16 \mathrm{MHz}$ Clock

5) Inputs and Outputs

6) digital Inputs/Outputs

7) 6 analog Inputs

8) 6 PWM-Outputs

\section{System Overview}

We have designed, developed, and deployed a multi-sensor network for monitoring landslide

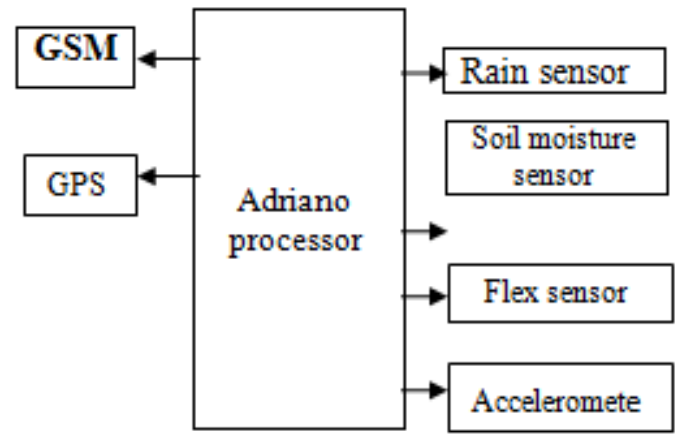

Figure 3: Block Diagram
The Field management unit will collect the necessary information from all the sensors. It also detects the position using GPS receiver. GPS receiver will give the latitude and longitude signals and we can detect the actual landslide location.

The sensor data collected with the location details will be transferred to the data management centre using GSM. The parameters which we want to measure are physical parameters (i.e in non -electrical form).so as to convert it into electrical form we are using sensors. The output of sensor is in analog in nature to convert it into digital form we apply it to ADC which is inbuilt in arduino[14]. The converted digital output of sensor is analyzed by controller for taking proper action. After that controller ask module it is ready or not. Then controller sends these data through UART to transceiver. The information is send to master module. It will receive it and decodes it. Then give it to Arduino controller and according to information is received, will display on in mobile SMS . Likewise information will be updated after every fixed duration. GSM will pass the information to every connected mobile.

\section{Result \& conclusion}

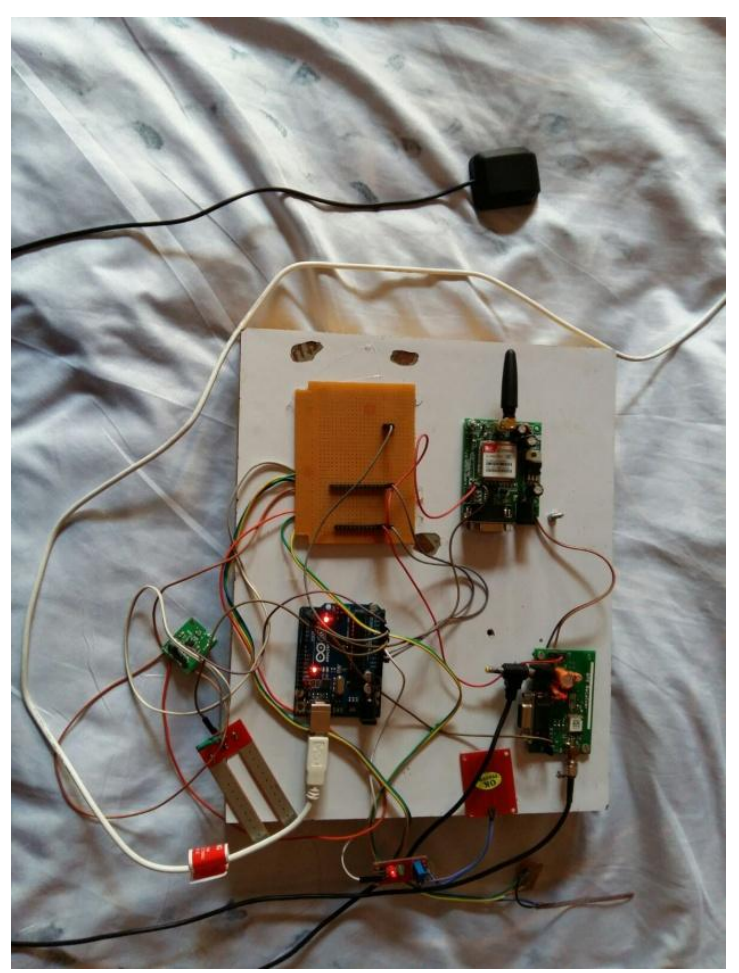

Figure 4: Hardwere connection

As the result all sensor sense continuously sensing rain,soil moisture, flex sensor, accelerometer sensor(both for movement).If heavy rain occur sms will send on mobile through GSM that "Landslide detected". GPS co-ordinate will send to aurdino processor. All sensor value will also given to aurduino. comparison will done with threshold values if heavy rainfall occur then message will send to save number in programming. This will help to local area people save life in case of landslide. 


\section{International Journal of Science and Research (IJSR) \\ ISSN (Online): 2319-7064}

Index Copernicus Value (2013): 6.14 | Impact Factor (2014): 5.611

Output screen:

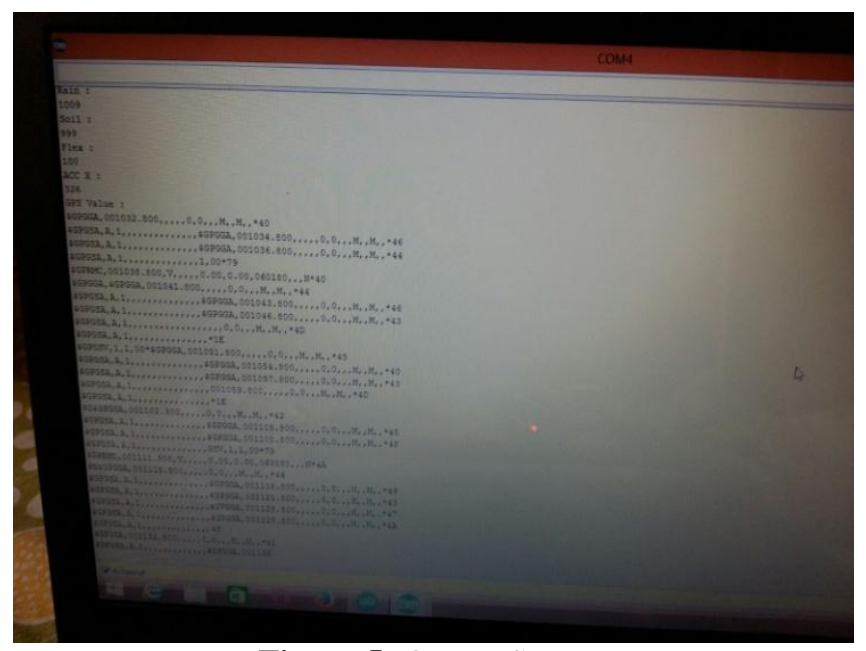

Figure 5: Output Screen
Wireless Opt. Commun. Netw. (WOCN), Sep. 2012, pp. $1-5$.

[11]N. Caine, "The rainfall intensity-duration control of shallow landslides and debris flows," Geografiska Annaler, vol. 62, nos. 1-2, pp. 23-27, 1980.

[12] M. V. Ramesh and N. Vasudevan, "The deployment of deep-earth sensor probes for landslide detection," Landslides, vol. 9, no. 4, pp. 457-474, 2012.

[13] M. V. Ramesh, "Design, development, and deployment of a wireless sensor network for detection of landslides," Ad Hoc Netw., vol. 13

[14] Andrew sloss,Dominic symes, chris wright,ARM system developer Guide.2004 Morgan kaufmann.ISBN 155860-874-5

\section{References}

[1] W. Ye, J. Heidemann, and D. Estrin, "An energyefficient MAC protocol for wireless sensor networks," in Proc. IEEE INFOCOM, vol. 3, Jun. 2002, pp. 15671576.

[2] T. He, B. M. Blum, J. A. Stankovic, and T. Abdelzaher, "AIDA: Adaptive application independent data aggregation in wireless sensor networks," ACM Trans. Embedded Comput. Syst., vol. 3, no. 2, pp. 426-457, May 2004.

[3] S. J. Baek, G. de Veciana, and X. Su, "Minimizing energy consumption in large-scale sensor networks through distributed data compression and hierarchical aggregation," IEEE J. Sel. Areas Commun., vol. 22, no. 6, pp. 1130-1140, Aug. 2004.

[4] A. Terzis, A. Anandarajah, K. Moore, and I.-J. Wang, "Slip surface localization in wireless sensor networks for landslide prediction," in Proc. IPSN, Apr. 2006, pp. 109-116.

[5] K. S. C. Kuang, S. T. Quek, and M. Maalej, "Remote flood 1monitoring system based on plastic optical fibres and wireless motes," Sens. Actuators A, Phys., vol. 147, no. 2, pp. 449-455, Oct. 2008.

[6] G. Werner-Allen, K. Lorincz, M. Welsh, O. Marcillo, J. Johnson, M. Ruiz, et al., "Deploying a wireless sensor network on an active volcano," IEEE Internet Comput., vol. 10, no. 2, pp. 18-25, Mar./Apr. 2006.

[7] M. Hefeeda and M. Bagheri, "Forest fire modeling and early detection using wireless sensor networks," Ad Hoc Sensor Wireless Netw., vol. 7, nos. 3-4, pp. 169-224, 2009.

[8] H.-Y. Kung, J.-S. Hua, and C.-T. Chen, "Drought forecast model and framework using wireless sensor networks," J. Inf. Sci. Eng., vol. 22, no. 4, pp. 751-769, Jul. 2006.

[9] E. R. Musaloiu, A. Terzis, K. Szlavecz, A. Szalay, J. Cogan, and J. Gray, "Life under your feet: A wireless soil ecology sensor network," in Proc. 3rd Workshop Embedded Netw. Sensors, May 2006, p. 481.

[10] K. A. Unnikrishna Menon, D. Maria, and T. Hemalatha, "Power optimization strategies for wireless sensor networks in coal mines," in Proc. 9th IEEE Int. Conf. 